Revue internationale P.M.E.

Économie et gestion de la petite et moyenne entreprise

Revure

internationale

PME

\title{
Les entrepreneurs artisans et opportunistes : Une comparaison de leurs comportements de gestion
}

\section{Jean Lorrain et Louis Dussault}

Volume 1, numéro 2, 1988

URI : https://id.erudit.org/iderudit/1007879ar

DOI : https://doi.org/10.7202/1007879ar

Aller au sommaire du numéro

Éditeur(s)

Presses de l’Université du Québec

ISSN

0776-5436 (imprimé)

1918-9699 (numérique)

Découvrir la revue

Citer cet article

Lorrain, J. \& Dussault, L. (1988). Les entrepreneurs artisans et opportunistes : Une comparaison de leurs comportements de gestion. Revue internationale P.M.E., 1(2), 157-176. https://doi.org/10.7202/1007879ar
Résumé de l'article

Cet article présente les résultats d'une étude sur les comportements de gestion privilégiés par les différents types d'entrepreneurs dont les entreprises manufacturières sont en phase de survie et d'établissement. Soixante-dix entrepreneurs ont répondu à des questionnaires sur les lieux de travail et les résultats révèlent que ces deux types d'entrepreneurs (entrepreneur artisan vs entrepreneur opportuniste) se différencient quant à leurs comportements de gestion à ce stade de développement de l'entreprise. La gestion de l'entrepreneur opportuniste apparaît plus équilibrée que celle de

l'entrepreneur artisan à ce stade de l'entreprise. Bref, la principale conclusion qui découle de cette recherche est l'importance de tenir compte des types d'entrepreneurs dans les études sur la gestion des PME à différents stades de leur existence. 


\title{
Les entrepreneurs artisans et opportunistes : Une comparaison de leurs comportements de gestion
}

\author{
Jean LORRAIN \\ Louis DUSSAULT \\ GREPME, Université du \\ Québec à Trois-Rivières
}

\begin{abstract}
RÉSUME
Cet article présente les résultats d'une étude sur les comportements de gestion privilégiés par les différents types d'entrepreneurs dont les entreprises manufacturières sont en phase de survie et d'établissement. Soixante-dix entrepreneurs ont répondu à des questionnaires sur les lieux de travail et les résultats révèlent que ces deux types d'entrepreneurs (entrepreneur artisan vs entrepreneur opportuniste) se différencient quant à leurs comportements de gestion à $œ$ stade de développement de l'entreprise. La gestion de l'entrepreneur opportuniste apparaît plus équilibrée que celle de l'entrepreneur artisan à ce stade de l'entreprise. Bref, la principale conclusion qui découle de cette recherche est l'importance de tenir compte des types d'entrepreneurs dans les études sur la gestion des PME à différents stades de leur existence.
\end{abstract}

\section{ABSTRACT}

This paper presents some results from a study about managerial behavior privileged by the various types of entrepreneurs whom manufacturing business are in the survival and establishment stage. A group of 70 entrepreneurs filled in questionnaires at their place of employment. The results reveal that these entrepreneurs may be divided in two groups (cratsman and opportunistic entrepreneurs) and that they are different because of their management behavior during this period. The management of the opportunistics entrepreneur appears to be more equilibrated than the craftman entrepreneur's. In brief, the main conclusion of this research is the importance to take into account the different types of entrepreneurs in the future studies on small businesses during their various stages of existence.

\section{RESUMEN}

Este articulo presenta los resultados de un estudio sobre los comportamientos de gestion privilegiados por los diferentes tipos de empresarios cuyas empresas manufactureras se encuentran en fase de sobrevivencia y de establecimiento. 70 empresarios respondieron cuestionarios en los lugares de trabajo y los resultados revelan que estos dos tipos de empresarios (empresario artesanal y empresario oportunista) se diferencian en cuanto a sus comportamientos de gestion a ese estado de desarrollo de la empresa. La gestion del empresario oportunista parece mas equilibrada que la del empresario artesanal a ese estado de la empresa. Breve, la principal conclusion que emerge de esta investigacion, es la importancia de tener cuenta de los tipos de empresarios en los estudios sobre la gestion de las PyME a los diferentes estados de su existencia 


\section{Introduction}

Les recherches dans le domaine de l'entrepreneuriat et de la PME s'inscrivent de plus en plus dans une perspective de contingence en ce sens qu'elles abordent cette problématique en spécifiant davantage les caractéristiques organisationnelles des PME, selon des critères reliés à la taille et à l'évolution, et les types d'entrepreneurs, selon des critères reliés à la motivation et aux antécédents personnels et professionnels de ceux-ci. Ceci favorise l'éclosion d'une nouvelle aire de recherche sur ces problématiques. Dans cet esprit, plusieurs chercheurs s'interrogent sur la relation entre les différents types d'entrepreneursetl'évolution de leurs entreprises (Sexton et Bowman, 1985; Stanworth et Curan, 1976; Churchill et Lewis, 1983). Même si les PME évoluent selon différents modes, d'aucuns nieront que le premier stade de ces entreprises est commun à tous. Certains l'appellent stade de survie et d'établissement (Gill, 1985) et d'autres, stade de démarrage («start up») (Fourcade, 1984). La plupart reconnaissent que ce stade est le plus critique pour l'entrepreneur et son entreprise. Sweenay (1983) intitule cette période de l'existence de la PME «la vallée de la mort», carc'est la période où le taux d'échec est le plus élevé pour les PME De plus, la survie des entreprises dépend principalement des entrepreneurs et de leur gestion. Il semble donc important de connaître les comportements de gestion de ceux-ci à cette phase.

\subsection{Comportements de gestion des entrepreneurs à la phase de survie et d'établissement}

Lesrecherches spécifiques sur les comportements de gestion des entrepreneurs à la phase de survie sont relativement rares. Seuls les modèles de développement des entreprises, en particulier les modèles «métamorphosis» abordent implicitement ce sujet (annexe 1, p. 170). En résumé, les informations contenues dans ces modèles concernant les comportements de gestion des entrepreneurs à ce stade révèlent que l'entrepreneur exécute une multitude de tâches de gestion et son principal objectif est la survie de son entreprise (Broom et alii, 1983; Churchill et Lewis, 1983; Hosmer et alii, 1977; Naumes, 1978; Quinn et Cameron, 1983; Steinmetz, 1969). Au niveau de la gestion managériale, l'entrepreneur planifie à court terme et d'une façon très informelle et très simpliste lorsqu'il s'implique dans cette activité de gestion (Churchill et Lewis, 1983; Naumes, 1978; Quinn et Cameron, 1983; Thain, 1969). Il centralise toutes les décisions (Chandler, 1967; Hosmer etalii, 1977); sa coordination est simple et très peu formalisée (Beckman et alii, 1982; Naumes, 1978; Quinn et Cameron, 1983) et sa communication avec ses employés est fréquente mais informelle (Greiner, 1972; Cannon, 1968). De plus, il contrôle les activités en se basant sur des critères personnels et subjectifs (Thain, 1969; Scott, 1970). Bref, les fonctions managériales à ce stade se caractérisent avant tout par leur simplicité et leur dimension informelle. Sur le plan de la gestion fonctionnelle, l'entrepreneur cherche à stabiliser sa production (Churchill et Lewis, 1983) et essaie de se constituer un 
créneau commercial (Basire, 1976; Naumes, 1978; Quinn et Cameron, 1983). Il s'implique directement dans la vente (Churchill et Lewis, 1983; Greiner, 1972) et délaisse souvent la comptabilité en la remettant à d'autres personnes (Buchele, 1967; Thain, 1969). De plus, les activités de recherche et développement et de gestion du personnel sont à caractère informel (Scott, 1970; Bechman et al., 1982).

Les modèles décrivent très simplement la gestion des entrepreneurs à ce stade de l'entreprise. A cet effet, Sexton et Bowman (1985) critiquent ces modèles parce qu'ils ne tiennent pas compte suffisamment des premiers stades fort importants de l'entreprise dans leur description de l'évolution des entreprises. De plus, ces modèles décrivent d'une façon très globale ce stade ainsi que les comportements des entrepreneurs. Pourtant, plusieurs auteurs de ces modèles signalent l'importance des caractéristiques des entrepreneurs sur la destinée et l'évolution de leur entreprise, entre autres Filley et Aldag (1978) et Churchill et Lewis (1983). Néanmoins, ils n'apportent ni attention ni distinction à l'égard des différents types d'entrepreneurs ainsi qu'à leurs comportements de gestion à ce stade important de leur entreprise.

\section{Les types d'entrepreneurs}

Dans la littérature en entrepreneuriat, différents auteurs présentent une typologie des entrepreneurs (annexe 2, p. 171). Smith (1967) fut l'un des premiers à présenter une typologie d'entrepreneurs bien articulée et reposant sur une base empirique. Elle comprend deux types d'entrepreneurs : l'entrepreneur artisan et l'entrepreneur opportuniste. L'entrepreneur artisan possède peu d'éducation mais une forte compétence technique. Le travail constitue le centre d'intérêt de cet entrepreneur et il adopte une attitude parternaliste au sein de son entreprise. Il craint de perdre le contrôle de son entreprise et refuse la croissance pour celle-ci. Pour sa part, l'opportuniste possède plus d'éducation et ses expériences de travail sont diversifiées et nombreuses. Cet entrepreneur s'identifie plus à la gestion et son attitude est non-paternaliste. Il ne refuse aucunement la croissance et ne craint pas la perte de contrôle de son entreprise.

A cette description sommaire de la typologie de Smith (1967), il faut ajouter celles de Stanworth et Curran (1976), de Filley et Aldag (1978), de Dunkelberg et Cooper (1984), de Gill (1985), de Laufer (1974), de Braden (1977) et de Ettinger (1982). Toutes ces typologies comportent de nombreuses différences en termes de méthodologie, de critères d'analyse et de nombre de typesd'entrepreneurs. Néanmoins, cette analyse fait ressortir quelques éléments communs. D'une part, on présente des caractéristiques personnelles et psychologiques (besoin d'indépendance/pouvoir, antécédents familiaux et professionnels s'associant à ces types d'entrepreneurs. D'autre part, on identifie les objectifs poursuivis et les comportements empruntés (désirabilité de croissance, professionnalisme de la gestion/structure) par ces types d'entrepreneurs. Par ailleurs, il faut reconnaître que la typologie de Filley et Aldag (1978) représente une typologie d'organisation et que certains auteurs l'utilisent à titre de typologie d'entrepreneurs. (Dunkelberg et Cooper, 1982; Vesper, 1982). 
Enfin, la typologie de Stanworth et Curran (1976) est la seule à présenter une dimension évolutive des types d'entrepreneurs. En ce sens, un entrepreneur peut changer de type compte tenu de la situation évolutive de la firme.

En résumé, l'ensemble des typologies nous amène à discerner deux groupes majeurs : les entrepreneurs artisans et les entrepreneurs opportunistes; et les éléments les différenciant doivent être perçus sous l'angle d'un continuum (ex : fort/faible désir de croissance d'une entreprise). De plus, il faut reconnaître que la description d'un type d'entrepreneur d'une typologie s'associe à l'occasion à celle d'un autre type d'entrepreneur d'une autre typologie (ex : Smith : «Craftman»; et Dunkelberg et Cooper : «craftmanship»). Toutefois, ces associations ne sont pas parfaites comme le soulignent Dunkelberg et Cooper (1982).

\section{Objectif de la recherche}

En résumé, les études ayant exploré les caractéristiques de gestion et les types d'entrepreneurs sont peu nombreuses. Par ailleurs, les nombreux travaux portant sur la gestion des PME et les types d'entrepreneurs ne nous permettent pas de connaître les comportements de gestion pouvant être privilégiés par chacun de ces types d'entrepreneurs lors du démarrage de l'entreprise. Lesmodèles de développement et les typologies d'entrepreneurs apportent certaines précisionsà cet égard. Cependant, on observe que peu de modèles de développement ont fait l'objet d'une vérification empirique et que les typologies d'entrepreneurs accordent peu d'attention à la description des comportements de gestion des entrepreneurs. Devant ces constats, l'objectif de cette recherche est d'examiner les comportements de gestion des différents types d'entrepreneurs dont l'entreprise est en phase de survie.

\section{$3 \quad$ Méthodologie}

\section{1 Échantillon}

L'échantillon de la présente recherche est composé de soixante-dix entrepreneurs (c'est-à-dire créateurs-propriétaires-dirigeants) ayant lancé une nouvelle entreprise dans le secteur manufacturier et ces entreprises sont localisées dans quatre régions du Québec. C'est à partir d'une banque de 800 entreprises du fichier central des entreprises du gouvernement du Québec que furent sélectionnées les entreprises. Les critères d'échantillonnage stipulaient qu'elles devaient être de nouvelles entreprises, ayant moins d'un an d'existence, dans le secteur manufacturier. Les critères utilisés afin de fractionner l'échantillon (entrepreneur artisan - entrepreneur 
opportuniste) sont le niveau d'éducation et les expériences antérieures de l'entrepreneur. Selon Smith (1967), les entrepreneurs opportunistes se distinguent principalement des entrepreneurs artisans par leur niveau de scolarité très élevé ainsi que leur expérience dans le domaine de la gestion. Le groupe des entrepreneurs artisans est composé de 41 entrepreneurs tandis que les entrepreneurs opportunistes sont au nombre de 29.

\subsection{Instrument de mesure}

Les comportements de gestion et les caractéristiques personnelles des entrepreneurs ont été mesurés par des questionnaires. Un instrument mesure les comportements de gestion administrative (planification, organisation, direction et contrôle) et fonctionnelle (production, marketing, finance, ressources humaines, recherche et développement) des entrepreneurs. Ce questionnaire a été spécialement conçu pour les fins de cette recherche. La consistance interne des échelles de cet instrument varie de 0,70 à 0,85 (alpha de Cronback). Un questionnaire comprenant des questions ouvertes et fermées a servi à mesurer les caractéristiques personnelles des entrepreneurs de même que certaines caractéristiques de leur entreprise.

\subsection{Procédure d'enquête}

La procédure d'enquête de cette recherche est la suivante : l'identification des nouvelles entreprises manufacturières à partir des listes du fichier central des entreprises et des informations obtenues des commissaires industriels, le contact téléphonique avec les entrepreneurs pour demander leur participation à la recherche, la présentation des questionnaires à remplir lors d'une rencontre individuelle sur le lieu de travail ainsi que la subdivision de notre échantillon en deux groupes : entrepreneurs artisans et entrepreneurs opportunistes.

\section{$4 \quad$ Présentation des résultats}

Les premiers résultats décrits sont ceux des caractéristiques personnelles des entrepreneurs artisans et opportunistes et des caractéristiques organisationnelles de leur entreprise. L'âge moyen des entrepreneurs artisans est de 32 ans et de 34,4 ans pour les opportunistes. Les entrepreneurs opportunistes ont en moyenne 15,5 années de scolarité et les artisans 11,2 années. De plus, $41,3 \%$ des entrepreneurs opportunistes affirment avoir suivi des cours de gestion avant de partir en affaires, comparativement à 29,2 \% pour les artisans. Par ailleurs, 73,2 \% des entrepreneurs artisans ont déjà travaillé dans une PME, comparativement à $44,8 \%$ pour les opportunistes. Par contre, les résultats obtenus indiquent que $72,4 \%$ des entrepreneurs 
opportunistes ont une expérience de gestion, en comparaison de seulement 19,5\% pour les artisans. De plus, $48,3 \%$ des opportunistes ont une expérience dans la grande entreprise, comparativement à $21,9 \%$ pour les entrepreneurs artisans. Ces premiers résultats montrent la validité du fractionnement de l'échantillon en entrepreneurs artisans et opportunistes, car le niveau de scolarité et les années d'expérience dans le domaine de la gestion du premier groupe sont moindres que dans le deuxième groupe. Il ressort également de ces résultats que les entrepreneurs artisans acquièrent davantage leur expérience de travail dans les PME tandis que les opportunistes la reçoivent dans les grandes entreprises.

Concernant les résultats des caractéristiques des entreprises des entrepreneurs artisans et opportunistes, l'âge moyen des firmes liées à l'entrepreneur artisan était de 4,78 mois et de 6,17 mois dans le cas de l'opportuniste. Par ailleurs, 82,9\% des entreprises liées aux entrepreneurs artisans sont des entreprises artisanales (c'est-àdire moins de quatre employés), comparativement à $44,8 \%$ dans le cas des opportunistes. La moyenne des employés réguliers au sein des entreprises des artisans est de 3,5 personnes et de 8,1 pour les opportunistes. Ce résultat inclut l'entrepreneur qui y travaille.

La forme légale «propriétaire-unique» est favorisée par les entrepreneurs artisans dans $60,9 \%$ des cas, tandis que $65,5 \%$ des entrepreneurs opportunistes privilégient la forme «compagnie avec actionnaires». Un conseil d'administration existe dans seulement $17 \%$ des entreprises dirigées par un entrepreneur artisan et la moyenne des participants à ce conseil est de 3,2 membres. Par contre, le conseil d'administration est présent dans $55,1 \%$ des entreprises dirigées par un entrepreneur opportuniste et la moyenne des participants est de 3,1 membres. Il existe un palier hiérarchique dans $43,9 \%$ des firmes de l'entrepreneur artisan, contre $82,7 \%$ dans les firmes de l'entrepreneur opportuniste. De plus, l'échelle de subordination est en moyenne de 2,8 personnes pour l'artisan et de 3,16 pour l'opportuniste. Alors que le type de production favorisé en majorité par les artisans $(74,6 \%)$ et par ceux opportunistes $(72,4 \%)$ est associé au carnet de commande. L'artisan est le directeur des opérations de l'entreprise dans $95,1 \%$ des cas, en comparaison de $79,3 \%$ pour l'opportuniste. L'ensemble des résultats sur les caractéristiques organisationnelles des entreprises révèle des différences importantes en termes de taille (nombre d'employés) et de structuration entre les entreprises des entrepreneurs opportunistes et celles des entrepreneurs artisans et ce, dès la création. Les opportunistes semblent démarrer de plus grandes entreprises que les artisans.

Les résultats présentés au tableau 1, (p. 163-164), font état de la présence de certaines caractéristiques de gestion des entrepreneurs artisans et opportunistes au sein de leur entreprise. Cesrésultats indiquent qu'une forte proportion desentrepreneurs artisans $(90,2 \%)$ et opportunistes $(100 \%)$ ont des objectifs généraux. Par contre, seulement $21,9 \%$ des artisans et $20,6 \%$ des opportunistes affirment avoir écrit ces objectifs. De plus, ces résultats indiquent qu'environ $30 \%$ des entrepreneurs artisans ou opportunistes ont élaboré des objectifs à long terme. La formulation d'objectif à caractère stratégique chez l'artisan est apparue dans $7,3 \%$ des cas, en comparaison 
de $13,7 \%$ chez l'opportuniste. La formulation d'objectifs à caractère personnel chez l'artisan est apparue dans $20,7 \%$ des cas et dans $13,7 \%$ chez les opportunistes. Par contre, la formulation d'objectifs liés à la fonction marketing chez l'entrepreneur opportuniste est apparue dans $25,8 \%$ des cas, en comparaison de $13,4 \%$ chez l'artisan. En somme, ces résultats tendent à démontrer le peu d'intérêt de ces entrepreneurs pour la planification stratégique à long terme.

Des plans formels ont été élaborés par les entrepreneurs opportunistes dans $68,9 \%$ des cas, en comparaison de $36,5 \%$ pour l'artisan. Ces plans formels touchent principalement la fonction production $(34,1 \%)$ dans le cas des artisans et la fonction production $(75,8 \%)$ et finance $(65,5 \%)$ dans le cas de l'opportuniste. Les résultats obtenus indiquent que l'entrepreneur opportuniste partage des tâches avec des partenaires dans $62,1 \%$ des cas, en comparaison de $31,7 \%$ pour l'artisan. De plus, ce partage s'effectue principalement au niveau de la fonction production $(51,7 \%)$ pour l'opportuniste et au niveau de la finance $(17,1 \%)$ et de la production $(17,1 \%)$ pour l'artisan. Malgré le fait que les entrepreneurs artisans et opportunistes ne formulent pas de stratégies à long terme, ils se distinguent néanmoins au niveau de la planification opérationnelle et de la répartition des responsabilités administratives.

Tab. 1. Caractéristiques de gestion des entrepreneurs artisans et opportunistes

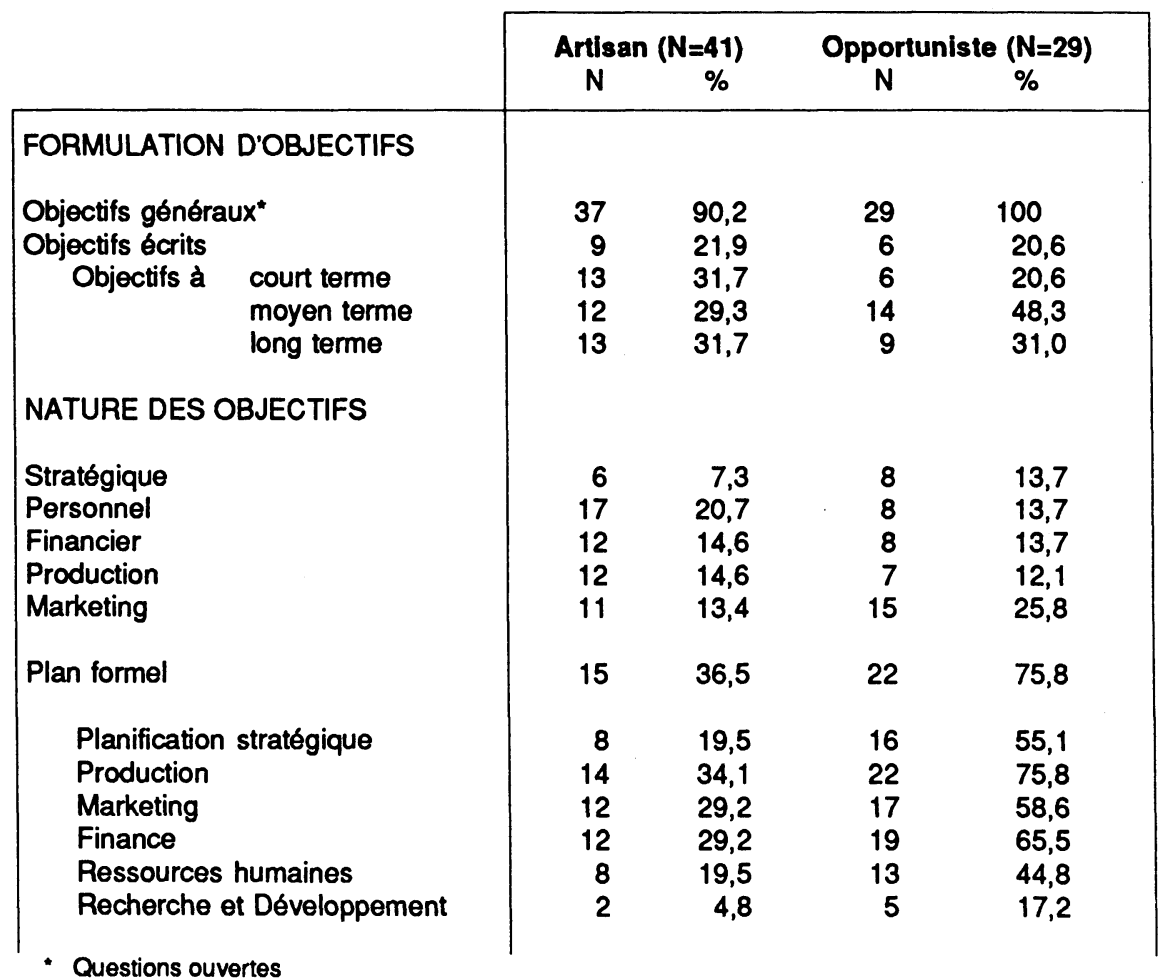


Tab. 1. (suite)

\begin{tabular}{|l|cccc|}
\cline { 2 - 5 } \multicolumn{1}{c|}{} & \multicolumn{3}{c}{ Artisan $(\mathbf{N}=\mathbf{4 1})$} & \multicolumn{2}{c|}{ Opportuniste $\mathbf{( N = 2 9 )}$} \\
\multicolumn{1}{c|}{} & $\mathbf{N}$ & $\mathbf{\%}$ & $\mathbf{N}$ & $\%$ \\
\hline TACHES DES PARTENAIRES & 13 & 31,7 & 18 & 62,1 \\
& & & & \\
Planification stratégique & 6 & 14,6 & 10 & 34,5 \\
Production & 7 & 17,1 & 15 & 51,7 \\
Marketing & 5 & 12,2 & 7 & 24,1 \\
Finance & 7 & 17,1 & 6 & 20,6 \\
Ressources humaines & 3 & 7,3 & 8 & 27,6 \\
Recherche et développement & 2 & 4,8 & 5 & 17,2 \\
\hline
\end{tabular}

Le tableau 2, (p. 165), présente des résultats globaux concernant les comportements de gestion des entrepreneurs artisans et opportunistes. Ces résultats font état de la présence des différentes fonctions au sein des firmes étudiées et de la moyenne d'utilisation de ces comportements de gestion par lesentrepreneurs artisans et opportunistes.

Les résultats indiquent que les activités de production, de marketing, de finance, de planification, d'organisation et de contrôle sont présentes dans presque toutes les firmes des entrepreneurs artisans et opportunistes, car leur pourcentage respectif varie de $96,5 \%$ à $100 \%$. Par ailleurs, les comportements de gestion les plus fréquemment utilisés par les entrepreneurs artisans et opportunistes sont liés aux fonctions production $(3,34-3,86)$ et direction $(3,17-3,76)$. Par contre, les comportements les moins utilisés sont associés à l'organisation $(2,04)$ et aux ressources humaines $(2,18)$ dans le cas de l'artisan et aux fonctions organisation $(2,86)$ et ressources humaines $(2,90)$ dans le cas de l'opportuniste. La moyenne obtenue par l'entrepreneur artisan à l'égard des comportements de gestion est de 2,73 et de 3,37 pour l'opportuniste.Le style comportemental de gestion de ces entrepreneurs est très semblable et ne les distingue d'aucune façon. Un fait intéressant est l'intérêt que portent ces entrepreneurs à la direction de leurs employés.

Un test U de Mann-Whitney a été effectué afin de déterminer s'il existe des différences significatives entre ces deux typesd'entrepreneurs. Cette analyse statistique non-paramétrique s'avère la plus appropriée pour déterminer les différences significatives étant donné que les comportements de gestion ont été mesurés par des échelles ordinales. Ces résultats révèlent que sur toutes les fonctions d'entreprises et managériales, sauf à la fonction direction, la valeur Uest significativement différente à un seuil de signification de 0,05 par rapportà celles des entrepreneurs artisans. Cinq pratiques de gestion différencient davantage ces deux types d'entrepreneur car le seuil de signification est inférieur à 0,01 : marketing, finance, planification, organisation et contrôle.L'annexe 3,(p. 172-176), nous présente les résultats de ce test concernant les différents énoncés composant les activités de gestion. 
Tab. 2 Résultats au test-U Mann Whitney sur les pratiques de gestion

\begin{tabular}{|c|c|c|c|c|c|c|c|c|c|c|c|}
\hline \multirow[b]{2}{*}{$\begin{array}{l}\text { Fonctions de } \\
\text { l'entreprise }\end{array}$} & \multicolumn{5}{|c|}{ Artisan } & \multicolumn{5}{|c|}{ Opportuniste } & \multirow[b]{2}{*}{ U/P } \\
\hline & $\mathbf{N}$ & $\bar{x}$ & o & MD & $R G$ & $N$ & $\bar{x}$ & o & MD & $R G$ & \\
\hline Production & 39 & 3,34 & 0,77 & 3,38 & 29,01 & 28 & 3,86 & 0,80 & 4,04 & 40,95 & $351,5 / 0,06$ \\
\hline $\begin{array}{l}\text { Marketing } \\
\text { Ressources }\end{array}$ & 39 & 3,89 & 0,77 & 2,81 & 26,37 & 28 & 3,58 & 0,73 & 3,69 & 44,63 & $248,5 / 0,00$ \\
\hline $\begin{array}{l}\text { humaines } \\
\text { Recherche \& }\end{array}$ & 27 & 2,18 & 0,95 & 2,10 & 20,83 & 24 & 3,90 & 0,85 & 3,20 & 31,81 & $184,5 / 0,04$ \\
\hline développement & 25 & 2,85 & 0,76 & 2,63 & 18,34 & 16 & 3,26 & 0,89 & 3,27 & 25,16 & $133,5 / 0,04$ \\
\hline Finance & 39 & 2,79 & 0,89 & 2,83 & 27,60 & 28 & 3,57 & 0,96 & 3,79 & 42,91 & $296,5 / 0,00$ \\
\hline \multicolumn{12}{|l|}{$\begin{array}{l}\text { Fonctions } \\
\text { managériales }\end{array}$} \\
\hline Planification & 39 & 2,61 & 0,71 & 2,60 & 26,91 & 28 & 3,28 & 0,73 & 3,33 & 43,88 & $269,5 / 0,00$ \\
\hline Organisation & 39 & 2,04 & 0,89 & 1,92 & 27,00 & 28 & 2,86 & 0,90 & 3,08 & 43,75 & $273,5 / 0,00$ \\
\hline Direction & 27 & 3,17 & 1,24 & 3,69 & 23,13 & 24 & 3,76 & 0,72 & 3,83 & 29,23 & $246,5 / 0,14$ \\
\hline Contrôle & 39 & 2,73 & 0,78 & 2,72 & 27,92 & 28 & 3,30 & 0,80 & 3,61 & 42,46 & $309,0 / 0,00$ \\
\hline Moyenne & \multicolumn{5}{|c|}{$(\bar{X}=2,73)$} & \multicolumn{5}{|c|}{$(\bar{x}=3,37)$} & \\
\hline
\end{tabular}




\section{$5 \quad$ Discussion et conclusion}

La présente étude révèle des résultats fort intéressants concernant les comportements de gestion des entrepreneurs artisans et opportunistes lorsque leur entreprise est en phase de survie et d'établissement. Ces résultats montrent que ces deux types d'entrepreneurs diffèrent quant à leur manière de gérer leur entreprise à ce stade. Par exemple, l'entrepreneur opportuniste s'implique beaucoup plus personnellement que l'entrepreneur artisan dans toutes les différentes fonctions de la gestion de son entreprise, sauf à la direction des ressources humaines. De plus, cet entrepreneur formule plus de plans et partage davantage les tâches administratives avec ses partenaires comparativement à l'entrepreneur artisan. Ces résultats montrent en somme que la gestion de l'entrepreneur opportuniste est plus équilibrée que celle de l'entrepreneur artisan à ce stade de l'entreprise. Par ses comportements, l'entrepreneur opportuniste se préoccupe de l'aspect administratif et opérationnel de la gestion tandis que l'entrepreneur artisan s'intéresse surtout à la dimension opérationnelle. Ceci peut expliquer les différences quant aux caractéristiques organisationnelles de la jeune entreprise dirigée par ces deux types d'entrepreneurs. L'entreprise de l'entrepreneur opportuniste comporte plus d'éléments de structuration, par exemple, la présence d'un conseil d'administration, et est souvent de plus grande taille (en termes du nombre d'employés) même si son entreprise n'est pas plus vieille que celle de l'entrepreneur artisan. Par conséquent, cet entrepreneur doit investir plus dans son entreprise. Ces résultats s'avèrent les plus intéressants car ils semblent montrer une différence importante concemant les entreprises créées par les entrepreneurs opportunistes et artisans en ce sens que les premiers créent une entreprise de plus grande taille et mieux structurée que les autres. Aucune recherche n'a, jusqu'à ce jour, signalé cette particularité de la création d'entreprise par ces deux types d'entrepreneurs.

Les différences quant à la gestion de l'entrepreneur opportuniste et de l'entrepreneur artisan peuvent s'expliquer également par les caractéristiques personnelles et psychologiques de ces entrepreneurs. L'entrepreneur opportuniste possède une plus grande expérience dans le domaine de la gestion avant de créer son entreprise, ce qui lui a sans doute permis de développer plus d'habiletés et de mieux connaître les techniques et les méthodes reliées à la gestion. Au contraire, l'entrepreneur artisan a moins d'expérience en gestion et par conséquent possède une compétence limitée dans ce domaine. De plus, les objectifs personnels poursuivis parl'entrepreneur opportuniste, habituellement en relation avec la croissance de l'entreprise, font que ce dernier doit s'impliquer dans tous les aspects de la gestion dès le lancement de son entreprise pour réaliser ses objectifs. L'entrepreneur artisan en poursuivant des objectifs de satisfaction personnelle s'implique moins dans la gestion de son entreprise à ce stade de son entreprise.

Ces résultats remettent en cause la validité et l'utilité de la description des modèles au stade de démarrage de l'entreprise. D'une part, les entrepreneurs gèrent différemment leur entreprise, ce qui aura des effets importants sur le mode et le 
rythme d'évolution de celle-ci. D'autre part, cette recherche montre la faiblesse des modèles au niveau de la description de la gestion des entrepreneurs à ce stade. Cette remarque rejoint les commentaires formulés à cet égard par Sexton et Bowman (1985). La gestion des entreprises lorsqu'elles sont au stade de survie est aussi plus complexe que ne le laissent prétendre les modèles et comme la réalité le rappelle bien. Sinon, comment expliquer le taux élevé d'échec des entrepreneurs à ce stade? La méthodologie utilisée dans la présente recherche et les études sur l'évolution des entreprises peuvent expliquer cet écart au niveau des résultats. Les modèles de développement reposent habituellement sur des recherches faites auprès d'entrepreneurs dont l'entreprise existe depuis plusieurs années et est parvenue à une phase organisationnelle avancée. Ce sont des recherches dites historiques où les entrepreneurs décrivent l'évolution de leur entreprise. Par conséquent, il se peut que ceux-ci ne se remémorent pas tous les éléments et leurs agissements du passé lorsqu'ils ont démarré leur entreprise et/ou rationalisé cette période en se préoccupant davantage de leur gestion au moment présent. Mais en investiguant leurs comportements précisément à ce stade, on découvre l'intensité et la complexité de leur gestion à cette période de l'entreprise.

Ces résultats sont aussi révélateurs quant à l'importance de tenir compte des types d'entrepreneur dans les modèles «métamorphosis« de l'évolution de l'entreprise et confirment la validité empirique d'une typologie d'entrepreneurs basée sur les caractéristiques personnelles de ceux-ci mais aussi sur leurs comportements de gestion. Dans ce contexte, les recherches futures sur l'évolution des PME devraient différencier les entrepreneurs lors de leur investigation empirique.

Cette recherche ne fait qu'éplucher la problématique des comportements de gestion des entrepreneurs aux différents stades de leur entreprise. Elle indique par contre son importance. Des études longitudinales devraient être entreprises pour étudier la relation entre les comportements de gestion des différents entrepreneurs, ainsi que leur évolution et le mode de croissance de leur entreprise et ce, dès le lancement de l'entreprise. Par contre, cela nécessite l'existence d'une typologie validée d'entrepreneurs. Devant la multiplicité de ces typologies, il s'avère important que les recherches futures sur cette problématique déterminent la validité de contenu en concomitance des typologies existantes pour identifier celle qui représente mieux cette réalité.

Pour terminer, il convient de souligner les principales limites de cette recherche. La principale est la taille de l'échantillon, ce qui a pour effet de restreindre la validité externe de cette recherche et par conséquent la portée de nos conclusions. Une autre limite est la méthode de classement des entrepreneurs, basée principalement sur deux critères, lesquels ont permis de diviser les entrepreneurs en deux groupes. Il serait souhaitable que les recherches futures prennent de plus grands échantillons et regroupent les entrepreneurs selon une typologie validée plus large. Malgré ces limites méthodologiques, il n'en demeure pas moins que cette recherche fournit un apport intéressant à l'explication des comportements de gestion des entrepreneurs opportunistes et artisans au stade de survie de l'entreprise. Elle pourrait même servir de point de départ pour répondre à ces questions. 


\section{BIBLIOGRAPHIE}

Basire, M., (1976) «La théorie des 5 niveaux», Direction et gestion, $n^{\circ} 2$, pp. 11-21, $n^{\circ} 3$, pp. 11-22, $n^{\circ} 4$, pp. 13-20, $n^{\circ} 5$, pp. 11-16.

Beckman, M.D., Good, W.S. et Wyekman, R.G., (1982), Small Business Management, Concepts and Cases, John Wiley and Sons Limited.

Braden, P., (1984), «Entrepreneurship and paths to business ownership», dans Dunkelberg, W.C., et A.C. Cooper, op. cité.

Broom, H.N., Longenecker, J.G. et Moore, C.W.,(1983), Small Business Management, SouthWestern Publishing Co., $6^{\circ}$ édition

Buchele, B.R., (1967), Business Policy in Growing Firms, Chaldler Publishing Company.

Cannon, J.T. (1968), dans Glueck, W.L. et Jauch, L.R., (1984), Business Policy and Strategic Management, McGraw-Hill, $4^{\circ}$ édition.

Chandler, A. Jr., (1967), dans Glueck, W.L. et Jauch, L.R., (1984), Op. cit.

Churchill, N.C. et Lewis, V.L., (1983), «Les cinq stades de l'évolution d'une P.M.E.», Harvard l'Expansion, automne, pp. 51-63.

Cooper, A.C., (1969), «Strategic Management : new ventures and small business», dans D.E. Schendel et C.W. Hofer, Strategic Management : A New View of Management Policy and Planning, Little Brown and Company.

Drucker, P.F., (1954), La pratique de la direction des affaires, Éditions d'Organisation, pp. 235-261.

Dunkelberg, W.C. et Cooper, A.C., dans Vesper, K.H., (1982), «Frontiers of entrepreneurship research», Proceedings of the conference on entrepreneurship.

Dunkelberg, W.C. et Cooper, A.C., (1984), Entrepreneurship and Pathsto Business Ownership, Purdue University, West Lafayette, Indiana.

Ettinger, J.C., (1982), «Some belgian evidence on entrepreneurial personality, European Small Business Journal, 1, 2, hiver.

Filley, A.C. et Aldag, R.J., (1978), «Characteristics and measurement of an organizational typology", Academy of Management Journal, vol. 21, $\mathrm{n}^{\circ} 4$, décembre, pp. 578-591.

Fortin, P.A., (1986), Devenez entrepreneur, Presses de l'Université Laval, Québec.

Fourcade, C., (1986), «Le démarrage des entreprises : comparaisons internationales» dans Julien, P.A. et alii (éds), LaPME en mutation, Presses de l'Université du Québec, Québec.

Gill, J., (1985), Factors Affecting the Survival and Growth of the Smaller Company, Gower Publishing Company.

Greiner, L.E., (1972), «Evolution and revolution as organization grow, Harvard Business Review, vol. 50, juillet-août, pp. 37-46.

Hosmer, L.T., Cooper, A.C. et Vesper, K.H., (1977), The Entrepreneurial Function, PrenticeHall, Englewood Cliffs, N.J.

Kroeger, C.R., (1974), «Managerial development in the small firm», California Management Review, vol XVIII, $\mathrm{n}^{\circ} 1$, automne, pp. 41-47.

Laufer, J., (1974), «Comment on devient entrepreneur», Revue Française de Gestion, 2. 
Lippitt, G.L. et Schmidt, W.H., (1967), «Crises in a developing organization», Harvard Business Review, vol. 45, $\mathrm{n}^{\circ}$ 6, pp. 102-112.

Naumes, W., (1978), The Entrepreneurial Management in the Small Business, AddisonWesley Publishing Company Inc.

Quinn, R.E. et Cameron, K., (1983), «Organization life cycles and shifting criteria of effectiveness : some preliminary evidence $\ll$,Management Science, vol. $29, \mathrm{n}^{\circ} 1$, janvier, pp. 33-51.

Robidoux, J., (1980), Les crises administratives dans les PME en croissance, Éditions Gaétan Morin, Chicoutimi.

Scott, B., (1970), dans Glueck, W.L. et Jauch, L.R., (1984), Business Policy and Strategic Management, McGraw-Hill Inc, $4^{\circ}$ édition.

Sexton, D.L. et Browman, N.B., (1985), «Growth orientation as a distinguishing factor between entrepreneurship and small business», Actes du colloque du CIPE, Montréal.

Siropolis, N.C., (1977), Small Business Management : A Guide to Entrepreneurship, Houghton Mifflin Company.

Smith, N.R., (1967), «The entrepreneur and his firm : the relationship between type of man and type of company», Bureau of Business and Economic Research, Michigan State University, East Lansing, Mich.

Stanworth, M.J.K. et Curran, J., (1976), «Growth \& small firm - an alternative view», Journal of Management Studies, vol. 13, $\mathrm{n}^{\circ} 2$, mai.

Steinmetz, L.L., (1969), «Critical stages of small business growth», Business Horizons, vol. XII, $n^{\circ} 1$, février, pp. 29-36.

Sweenay, G.P., (1982), Les nouveaux entrepreneurs : petites entreprises innovatrices, Éditions d'Organisation.

Tate, C.E., Megginson, L.C., Scoth, C.R. et Trueblood, L.R., Successful Small Business Management, $3^{\mathrm{e}}$ édition, Business Publications Inc.

Thain, D.H., (1969), «Stages of corporate development», The Business Quarterly, hiver, vol. $34, n^{\circ} 4$, pp. $32-45$.

Vargas, G., (1984), «Les crises de croissance de la PMI-PME», Revue Française de Gestion, janv.-fév., $\mathrm{n}^{\circ} 44$. 


\section{Annexe 1}

\section{Modéles de développement}

\begin{tabular}{|c|c|c|c|}
\hline & AUTEURS & NOMBRE DE STADES & PARAMETRES SPÉCIFIQUES \\
\hline & $\begin{array}{l}\text { Chandler (1967) } \\
\text { Lippitt et Schmidt (1967) } \\
\text { Scott (1970) } \\
\text { Thain (1976) } \\
\text { Cooper (1979) } \\
\text { Tate et al., (1972) }\end{array}$ & 3 & $\begin{array}{l}\text { Structure/stratégie } \\
\text { Crises organisationnelles } \\
\text { Gestion/Structure } \\
\text { Gestion/Fonctions clefs } \\
\text { Stratégie/Structure } \\
\text { Gestion/Structure }\end{array}$ \\
\hline & $\begin{array}{l}\text { Drucker (1954) } \\
\text { Steinmetz (1969) } \\
\text { Siropolis (1977) } \\
\text { Hosmer et al. (1977) } \\
\text { Naumes (1978) } \\
\text { Quinn et Cameron (1983) } \\
\text { Broom et al. (1983) } \\
\text { Fortin (1986) }\end{array}$ & 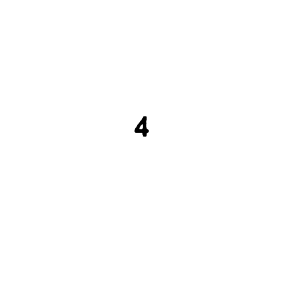 & $\begin{array}{l}\text { Situationnel/Structure } \\
3 \text { phases critiques/Rôles } \\
\text { situationnels } \\
\text { Rôles managériaux } \\
\text { Structure/Organisation } \\
\text { Structure/Organisation } \\
\text { Rôles managériaux } \\
\text { Situationnel/gestion }\end{array}$ \\
\hline $\begin{array}{l}* \\
\text { * } \\
\text { * }\end{array}$ & $\begin{array}{l}\text { Cannon (1968) } \\
\text { Greiner (1972) } \\
\text { Kroeger (1974) } \\
\text { Basire (1976) } \\
\text { Beckman et al. (1982) } \\
\text { Churchill et Lewis (1983) } \\
\text { Vargas (1984) } \\
\text { Gill (1985) }\end{array}$ & 5 & $\begin{array}{l}\text { Gestion/Structure } \\
\text { Crises administratives } \\
\text { Rôles managériaux } \\
\text { Gestion/Structure } \\
\text { Gestion/Organisation } \\
\text { Crises administratives } \\
3 \text { sous-systèmes } \\
\text { Situationnel/gestion }\end{array}$ \\
\hline & $\begin{array}{l}\text { Buchele (1967) } \\
\text { Robidoux (1980) }\end{array}$ & 7 & $\begin{array}{l}\text { Phases critiques de } \\
\text { croissance } \\
\text { Crises administratives }\end{array}$ \\
\hline ** & Parks (1977) & 11 & «Hurdles" \\
\hline
\end{tabular}

* Modèles qui semblent avoir fait l'objet d'une vérification empirique. Cependant, nous avons peu d'informations méthodologiques et peu de travaux de contre-vérification de ces différents modèles.

** Parks précise que les 11 uhurdles" présentés ne constituent pas l'ordre séquentiel pouvant être rencontré par un manager. 
Annexe 2

\section{Les typologies d'entrepreneur}

\begin{tabular}{|c|c|c|c|}
\hline Auteurs & Nombre & Type d'entrepreneur & Variable d'analyse \\
\hline Smith (1967) & 2 & $\begin{array}{l}\text { "Craftman" } \\
\text { "Opportunistic" }\end{array}$ & $\begin{array}{l}\text { - Entrepreneur } \\
\text { - Entreprise }\end{array}$ \\
\hline Braden (1977) & 2 & $\begin{array}{l}\text { "Caretaker" } \\
\text { «Manager" }\end{array}$ & $\begin{array}{l}\text { Motivation de } \\
\text { l'entrepreneur }\end{array}$ \\
\hline $\begin{array}{l}\text { Filley et } \\
\text { Aldag }(1962,1974 \\
1975)\end{array}$ & 4 & $\begin{array}{l}\text { ¿Craftsman" } \\
\text { "Promotion" } \\
\text { "Administration" }\end{array}$ & $\begin{array}{l}\text { - } \quad \text { Structure } \\
\text { - } \quad \text { Leadership }\end{array}$ \\
\hline Gill (1985) & 4 & $\begin{array}{l}\text { «Achiver" } \\
\text { "Bloker" } \\
\text { "Survey" } \\
\text { «Failure" }\end{array}$ & $\begin{array}{l}\text { - Motivations } \\
\text { - Degré de compétence }\end{array}$ \\
\hline Laufer (1974) & 4 & $\begin{array}{l}\text { Manager/lnnovateur } \\
\text { Prop./croissance } \\
\text { Prop./non-crois. } \\
\text { Artisan }\end{array}$ & $\begin{array}{ll}- & \text { Motivations } \\
\text { psychologiques } \\
\text { - } & \text { Comportements } \\
& \text { économiques }\end{array}$ \\
\hline $\begin{array}{l}\text { Dunkelgerg et } \\
\text { Cooper (1982) }\end{array}$ & 3 & $\begin{array}{l}\text { "Growth" } \\
\text { "Independence" } \\
\text { "Craftsmanship" }\end{array}$ & $\begin{array}{ll}- & \text { Objectifs } \\
\text { - } & \text { Traits } \\
\text { - } & \text { Organisation }\end{array}$ \\
\hline Ettinger (1982) & 2 & $\begin{array}{l}\text { Pouvoir } \\
\text { Indépendance }\end{array}$ & $\begin{array}{ll}\text { - } & \text { Pouvoir } \\
\text { - } & \text { Indépendance } \\
\text { - } & \text { Type de firme }\end{array}$ \\
\hline $\begin{array}{l}\text { Stanworth et } \\
\text { Curan (1976) }\end{array}$ & 3 & $\begin{array}{l}\text { "Artisan" } \\
\text { "Classical" } \\
\text { "Manager" }\end{array}$ & 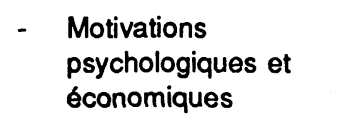 \\
\hline
\end{tabular}


Annexe 3

Détails des proportions des entrepreneurs artisans et opportunistes utilisant les pratiques de gestion

\begin{tabular}{|c|c|c|c|c|c|c|c|c|c|c|c|}
\hline \multirow[b]{3}{*}{ PRODUCTION } & \multicolumn{5}{|c|}{ ARTISAN } & \multicolumn{5}{|c|}{ OPPORTUNISTE } & \multirow[b]{2}{*}{$U / F$} \\
\hline & $\mathbf{N}$ & $x$ & $\boldsymbol{\sigma}$ & MD & $R G$ & $\mathbf{N}$ & $x$ & $\sigma$ & MD & RG & \\
\hline & & & & & & & & & & & \\
\hline 1. Objectifs de production & 39 & 3,69 & 1,47 & 4,0 & 30,85 & 28 & 4,29 & 1,18 & 5,0 & 38,39 & $423,0 / 0,09$ \\
\hline travail & 39 & 3,12 & 1,42 & 3,0 & 28,86 & 28 & 4,04 & 1,14 & 4,5 & 41,16 & $345,5 / 0,01$ \\
\hline 3. Utilisation maximale/ & & & & & & & & & & & \\
\hline $\begin{array}{l}\text { équipement } \\
\text { 4. Fluctuation des pertes }\end{array}$ & 39 & 3,21 & 1,59 & 3,0 & 31,64 & 28 & 3,68 & 1,42 & 4,0 & 37,29 & $454,0 / 0,23$ \\
\hline $\begin{array}{l}\text { de temps } \\
5 . \quad \text { Besoins en matières }\end{array}$ & 39 & 2,10 & 1,47 & 1,0 & 30,29 & 28 & 2,86 & 1,70 & 3,0 & 39,16 & $401,5 / 0,05$ \\
\hline premières & 39 & 3,28 & 1,57 & 3,0 & 30,95 & 28 & 3,86 & 1,53 & 4,5 & 38,25 & $427,0 / 0,11$ \\
\hline 6. Utilisation des ressources & 39 & 3,13 & 1,19 & 3,0 & 28,67 & 28 & 3,93 & 1,18 & 4,0 & 41,43 & $338,0 / 0,01$ \\
\hline $\begin{array}{l}\text { 7. Niveau des inventaires } \\
\text { 8. Sources d'approvision- }\end{array}$ & 39 & 2,82 & 1,71 & 3,0 & 31,32 & 28 & 3,43 & 1,73 & 4,0 & 37,73 & $441,5 / 0,16$ \\
\hline $\begin{array}{l}\text { nement } \\
\text { 9. Coûts et pertes/ }\end{array}$ & 39 & 3,31 & 1,54 & 4,0 & 31,58 & 28 & 3,68 & 1,72 & 5,0 & 37,38 & $451,5 / 0,21$ \\
\hline $\begin{array}{l}\text { production } \\
\text { 10. Qualité des produits }\end{array}$ & 39 & 3,23 & 1,55 & 3,0 & 32,50 & 28 & 3,54 & 1,48 & 4,0 & 36,09 & $487,5 / 0,44$ \\
\hline fabriqués & 39 & 4,72 &, 79 & 5,0 & 34,35 & 28 & 4,71 &, 66 & 5,0 & 33,52 & $532,5 / 0,79$ \\
\hline 11. Plaintes de clients & 39 & 3,56 & 1,80 & 5,0 & 31,06 & 28 & 4,21 & 1,55 & 5,0 & 38,09 & $431,5 / 0,09$ \\
\hline 12. Prix de revient & 39 & 3,70 & 1,44 & 4,0 & 30,96 & 28 & 4,21 & 1,32 & 5,0 & 38,23 & $427,5 / 0,10$ \\
\hline 13. Normes d'efficacité & 39 & 3,56 & 1,62 & 4,0 & 33,33 & 28 & 3,79 & 1,37 & 4,0 & 34,93 & $520,0 / 0,73$ \\
\hline
\end{tabular}




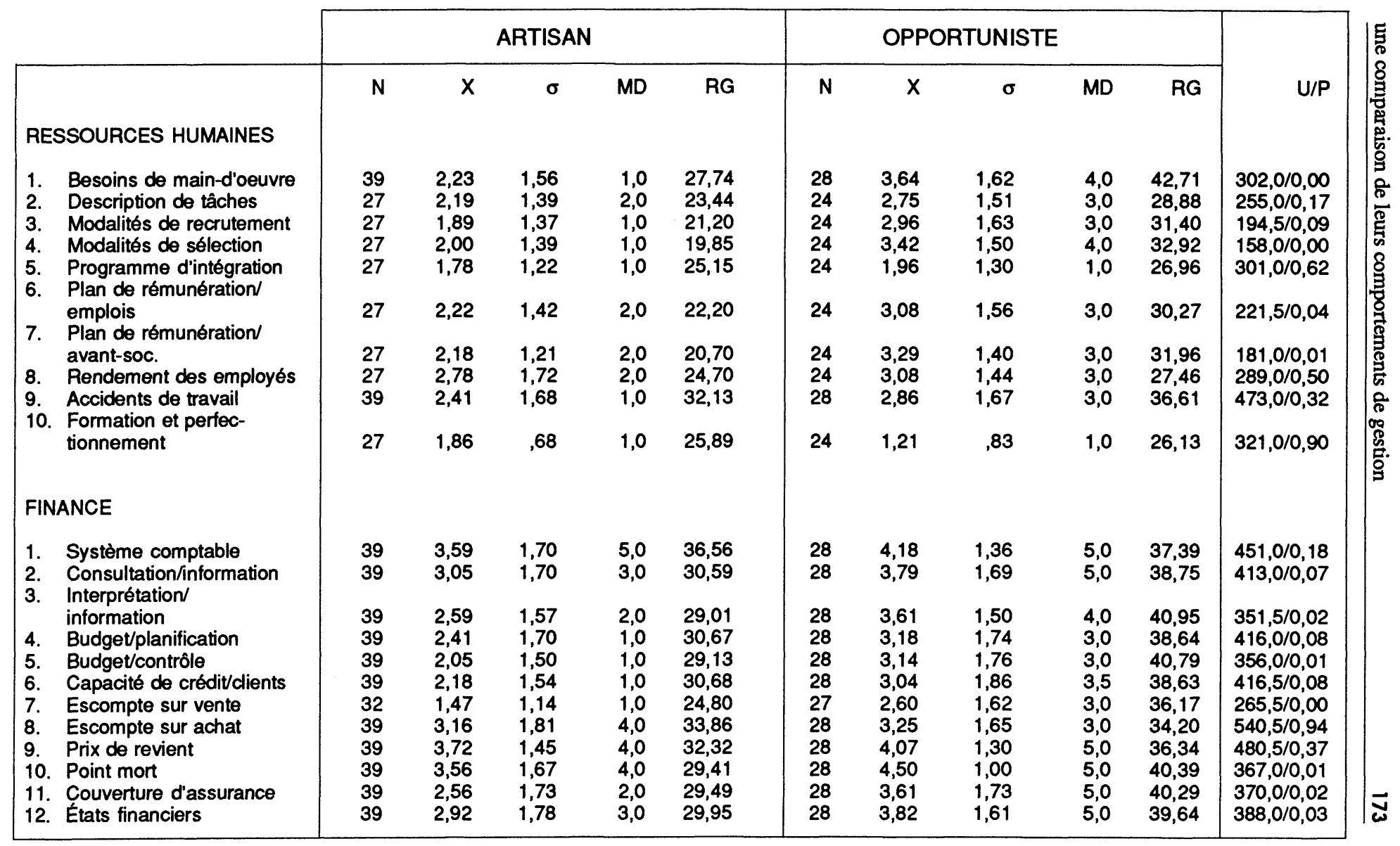




\begin{tabular}{|c|c|c|c|c|c|c|c|c|c|c|c|}
\hline \multirow[b]{3}{*}{ MARKETING } & \multicolumn{5}{|c|}{ ARTISAN } & \multicolumn{5}{|c|}{ OPPORTUNISTE } & \multirow[b]{2}{*}{$\mathrm{U} / \mathrm{F}$} \\
\hline & $\mathbf{N}$ & $x$ & $\sigma$ & MD & $R G$ & $N$ & $x$ & $\sigma$ & MD & $R G$ & \\
\hline & & & & & & & & & & & \\
\hline 1. Le marché & 39 & 3,69 & 1,28 & 4,0 & 29,64 & 28 & 4,32 & 1,09 & 5,0 & 40,07 & $376,0 / 0,02$ \\
\hline 2. Nouveaux marchés & 39 & 2,97 & 1,51 & 3,0 & 29,22 & 28 & 3,82 & 1,63 & 5,0 & 40,66 & $359,5 / 0,01$ \\
\hline $\begin{array}{l}\text { 3. Besoins des clients } \\
\text { 4. Habitudes d'achats/ }\end{array}$ & 39 & 4,03 & 1,31 & 5,0 & 31,23 & 28 & 4,50 &, 92 & 5,0 & 37,86 & $438,0 / 0,11$ \\
\hline clients & 39 & 3,64 & 1,71 & 5,0 & 33,38 & 28 & 4,00 & 1,16 & 4,0 & 34,86 & $522,0 / 0,74$ \\
\hline 5. Les concurrents & 39 & 3,62 & 1,35 & 4,0 & 29,46 & 28 & 4,29 & 1,30 & 5,0 & 40,32 & $369,0 / 0,02$ \\
\hline 6. Part de marché & 39 & 1,62 & 1,22 & 1,0 & 31,18 & 28 & 2,04 & 1,32 & 1,0 & 37,93 & $436,0 / 0,10$ \\
\hline $\begin{array}{l}\text { 7. Objectifs marketing } \\
\text { 8. Objectifs marketing/ }\end{array}$ & 39 & 2,80 & 1,47 & 3,0 & 27,78 & 28 & 3,96 & 1,35 & 4,0 & 42,66 & $303,5 / 0,00$ \\
\hline concurrents & 39 & 2,64 & 1,51 & 3,0 & 27,08 & 28 & 4,00 & 1,47 & 5,0 & 43,64 & $276,0 / 0,00$ \\
\hline 9. Plans marketing & 39 & 2,46 & 1,54 & 2,0 & 28,81 & 28 & 3,54 & 1,53 & 4,0 & 41,23 & $343,5 / 0,01$ \\
\hline $\begin{array}{l}\text { 10. Recueillir de l'information } \\
\text { 11. Volume des ventes/ }\end{array}$ & 39 & 3,95 & 1,23 & 4,0 & 31,19 & 28 & 4,36 & 1,03 & 5,0 & 37,91 & $436,5 / 0,13$ \\
\hline produits & 39 & 2,60 & 1,45 & 3,0 & 28,54 & 28 & 3,71 & 1,68 & 5,0 & 41,61 & $333,0 / 0,00$ \\
\hline $\begin{array}{l}\text { 12. Dépenses des ventes } \\
\text { 13. Pénétration de nouveaux }\end{array}$ & 39 & 2,24 & 1,60 & 1,0 & 29,25 & 28 & 3,11 & 1,60 & 3,0 & 39,27 & $370,5 / 0,03$ \\
\hline produits & 39 & 3,00 & 1,34 & 3,0 & 32,42 & 28 & 3,29 & 1,65 & 4,0 & 36,20 & $484,5 / 0,42$ \\
\hline 14. Prix de revient & 39 & 3,31 & 1,36 & 4,0 & 28,40 & 28 & 4,14 & 1,38 & 5,0 & 41,80 & $327,5 / 0,00$ \\
\hline 15. Publicité & 39 & 1,74 & 1,02 & 1,0 & 32,99 & 28 & 2,00 & 1,36 & 1,0 & 35,41 & $506,5 / 0,58$ \\
\hline 16. Rendement/publicité & 39 & 1,97 & 1,41 & 1,0 & 32,64 & 28 & 2,18 & 1,44 & 1,5 & 35,89 & $493,0 / 0,45$ \\
\hline
\end{tabular}




\begin{tabular}{|c|c|c|c|c|c|c|c|c|c|c|c|}
\hline \multirow[b]{2}{*}{ DIRECTION } & \multicolumn{5}{|c|}{ ARTISAN } & \multicolumn{5}{|c|}{ OPPORTUNISTE } & \multirow[b]{2}{*}{$\mathrm{U} / \mathrm{P}$} \\
\hline & $\mathrm{N}$ & $x$ & $\sigma$ & MD & RG & $\mathbf{N}$ & $x$ & $\sigma$ & MD & RG & \\
\hline 1. Bonne relation/employés & 27 & 3,70 & 1,73 & 5,0 & 23,02 & 24 & 4,58 & ,93 & 5,0 & 29,35 & $243,5 / 0,07$ \\
\hline 2. Accomplir le travail & 27 & 3,52 & 1,72 & 5,0 & 29,85 & 24 & 2,50 & 1,53 & 2,0 & 21,67 & $220,0 / 0,04$ \\
\hline d'efforts & 27 & 3,56 & 1,78 & 5,0 & 24,07 & 24 & 4,17 & 1,27 & 5,0 & 28,17 & $272,0 / 0,27$ \\
\hline 4. Problèmes au travail & 27 & 3,70 & 1,83 & 5,0 & 24,06 & 24 & 4,38 & 1,28 & 5,0 & 28,19 & $271,5 / 0,23$ \\
\hline $\begin{array}{l}\text { 5. Attentes des employés } \\
6\end{array}$ & 27 & 3,07 & 1,59 & 3,0 & 23,63 & 24 & 3,58 & 1,64 & 4,0 & 28,67 & $260,0 / 0,21$ \\
\hline $\begin{array}{l}\text { employés } \\
\text { 7. Participation aux }\end{array}$ & 27 & 2,82 & 1,80 & 3,0 & 23,13 & 24 & 3,63 & 1,47 & 4,0 & 29,23 & $246,5 / 0,13$ \\
\hline décision/employés & 27 & 2,82 & 1,67 & 3,0 & 23,13 & 24 & 3,50 & 1,29 & 3,5 & 29,23 & $246,5 / 0,13$ \\
\hline $\begin{array}{l}\text { 8. Informations pertinentes } \\
\text { 9. Questions relatives au }\end{array}$ & 27 & 3,48 & 1,67 & 4,0 & 24,57 & 24 & 4,00 & 1,10 & 4,0 & 27,60 & $285,5 / 0,44$ \\
\hline $\begin{array}{l}\text { travail } \\
\text { 10. Plaintes et problèmes }\end{array}$ & 27 & 3,52 & 1,78 & 4,0 & 24,98 & 24 & 3,96 & 1,27 & 4,5 & 27,15 & $296,5 / 0,58$ \\
\hline au travail & 27 & 2,81 & 1,80 & 3,0 & 23,44 & 24 & 2,54 & 1,64 & 4,0 & 28,88 & $255,0 / 0,17$ \\
\hline 11. Idées et opinions/empl. & 27 & 3,44 & 1,83 & 5,0 & 23,22 & 24 & 4,29 & 1,20 & 5,0 & 29,13 & $249,0 / 0,11$ \\
\hline 12. «Feedback»/employés & 27 & 3,48 & 1,70 & 4,0 & 22,52 & 24 & 4,38 & 1,10 & 5,0 & 29,92 & $230,0 / 0,05$ \\
\hline $\begin{array}{l}\text { 13. Motivation } \\
\text { 14. Travail/comoétences }\end{array}$ & 27 & 3,52 & 1,76 & 4,0 & 22,07 & 24 & 4,58 &, 93 & 5,0 & 30,42 & $218,0 / 0,02$ \\
\hline de l'employé & 27 & 3,37 & 1,86 & 5,0 & 23,31 & 24 & 4,21 & 1,32 & 5,0 & 29,02 & $251,5 / 0,12$ \\
\hline $\begin{array}{l}\text { 15. Conflits entre employés } \\
\text { 16. Collaboration entre }\end{array}$ & 17 & 1,82 & 1,59 & 1,0 & 17,50 & 22 & 2,41 & 1,65 & 1,5 & 21,93 & $144,4 / 0,17$ \\
\hline employés & 17 & 4,12 & 1,54 & 5,0 & 19,35 & 22 & 4,50 & 80 & 5,0 & 20,50 & $176,0 / 0,71$ \\
\hline 18. Travail/intérêt de & 27 & 2,11 & 1,58 & 1,0 & 24,33 & 24 & 2,42 & 1,56 & 2,0 & 27,88 & $279,0 / 0,36$ \\
\hline vos employés & 27 & 2,63 & 1,71 & 2,0 & 23,50 & 24 & 3,25 & 1,68 & 3,5 & 28,81 & $256,5 / 0,18$ \\
\hline
\end{tabular}




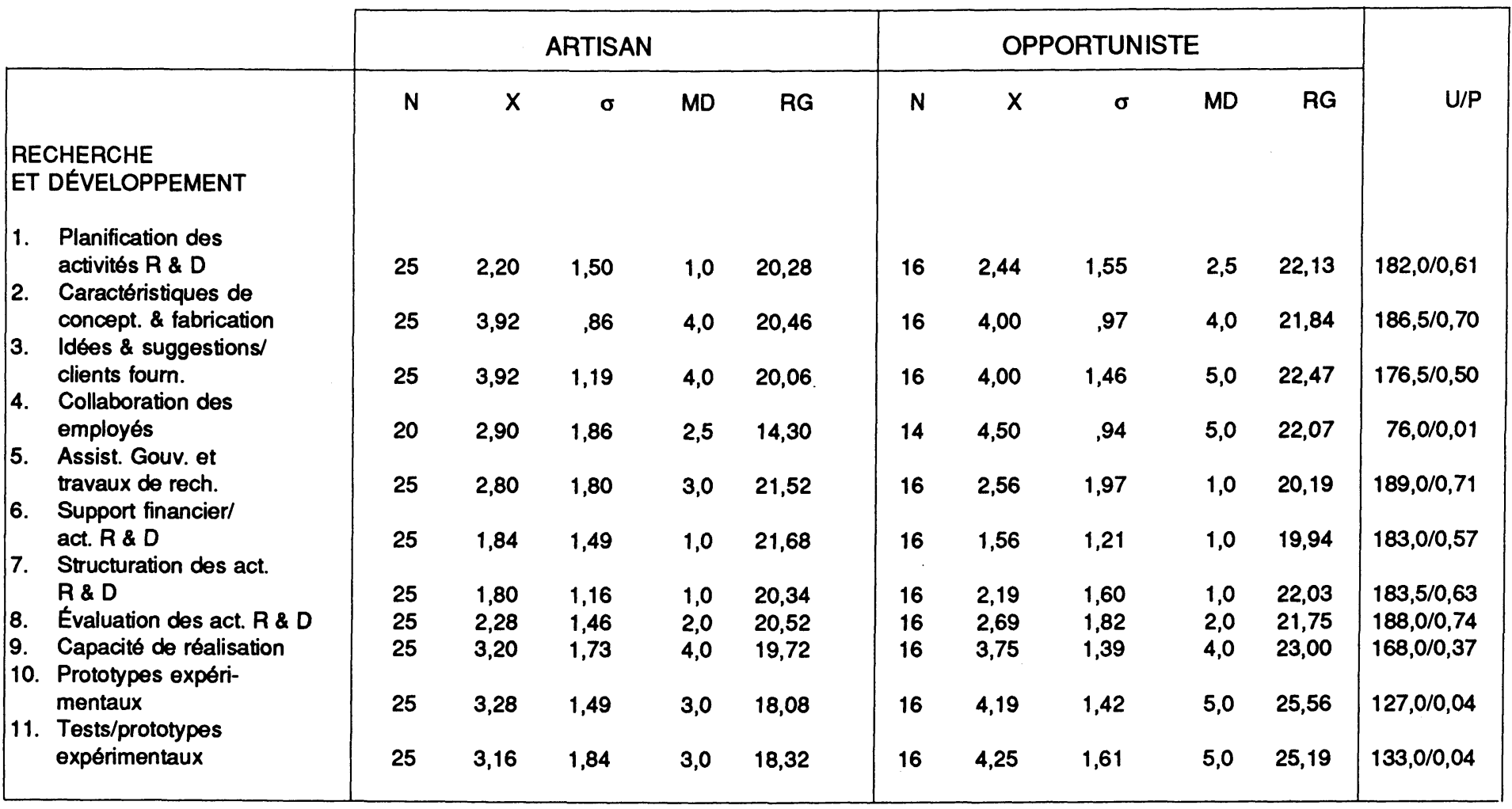

